\title{
Mitochondria and apoptosis: a quick take on a long view
} Melissa J Parsons and Douglas R Green*

\author{
Address: Department of Immunology, St Jude Children's Research Hospital, 262 Danny Thomas Place, Memphis, TN 38105, USA \\ *Corresponding author: Douglas R Green (douglas.green@stjude.org) \\ FI000 Biology Reports 2009, I:I7 (doi: 10.3410/BI-17)
}

The electronic version of this article is the complete one and can be found at: http://FI000.com/Reports/Biology/content/I/I7

\begin{abstract}
Fifteen years of apoptosis research have led to the widely accepted idea that the major form of programmed cell death in mammals proceeds via the mitochondria, and that mitochondrial control of apoptosis is regulated by a specialized family of proteins known as the Bcl-2 family. Here we will consider some very recent data that has shed new insight into the regulation of these proteins and the impact of mitochondrial dynamics on mitochondrial outer membrane permeabilization (MOMP) and apoptosis.
\end{abstract}

\section{Introduction and context}

It is always good to take the long view when considering recent history. Some 4 to 5 billion years ago, the earth formed, and almost as soon as it cooled about 500 million years later, life emerged. For another 2 billion years, the most toxic antagonist to life, oxygen, accumulated in the atmosphere until it reached the levels we face today. Bacteria evolved the trick of harnessing oxygen, and eventually, about 1 billion years ago, an $\alpha$-purple bacterium formed a symbiosis with another protist, an archeon, to create the first eukaryotic cell. The bacteria persist in us eukaryotes as thread granules (mitochondria) and service our cells with oxidative energy, lipid metabolism, the urea cycle, and oxygen detoxification. In us warmish creatures, they also provide heat, all of which, to paraphrase Bill Bryson, is nifty [1]. But 15 years ago, we first learned that these 'little tools' (organelles) have a dark side, playing a fundamental role in the predominant form of cell death in animals, known as apoptosis. The mitochondrial pathway of apoptosis, which concerns us here, exists at least throughout the vertebrates, and therefore this 'dark side' began almost 400 million years ago. In this brief overview, we concern ourselves with less than 1 year and our recent progress in understanding this mitochondrial function over the past few months (Figure 1).

\section{Major recent advances}

In the mitochondrial pathway of apoptosis, proteins in the Bcl-2 family function, primarily, to control the integrity of the outer mitochondrial membrane [2]. Two proteins, the proapoptotic effectors Bax and Bak, act to cause mitochondrial outer membrane permeabilization (MOMP), resulting in the diffusion of proteins of the intermembrane space (between the inner and outer mitochondrial membranes) into the cytosol, where one of these, cytochrome c, triggers a biochemical cascade resulting in the activation of caspase proteases and apoptotic cell death. Antiapoptotic members of the family, including Bcl-2, Bcl- $\mathrm{x}_{\mathrm{L}}, \mathrm{Mcl}-1$, and A1 (Figure 1), prevent MOMP and thereby prevent cell death. Another subfamily, consisting of the BH3-only proteins, so called because they share only the third Bcl-2 homology (BH) region, regulates the other two types by neutralizing the antiapoptotic proteins and/or activating the effectors to promote MOMP.

Several recent papers give new insights into this process. Gavathiotis et al. [3] used a number of nuclear magnetic resonance (NMR) techniques to provide a snapshot of how the BH3 region of Bim, which is one of the BH3only proteins that is thought to possess effector-activating activity, binds transiently to Bax to induce its activation. Bim appears to bind to a region of Bax opposite the 'BH groove' where, on the structurally related antiapoptotic proteins, $\mathrm{BH} 3$ domains normally bind. This somehow leads to oligomerization of Bax as it inserts into and disrupts the membrane. How this might happen comes from a study by Dewson et al. [4], who 
Figure I. The cast of characters

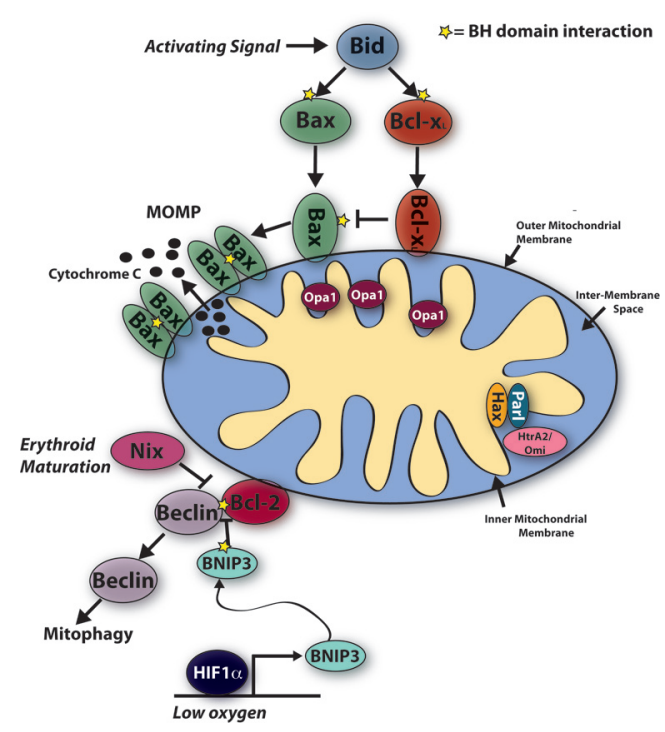

Shown are the location of mitochondrial 'characters' and a subset of their proposed functions in the regulation of mitochondrial apoptosis and mitophagy as discussed in the text. (Top) Upon receiving an activating signal, a direct activator $\mathrm{BH} 3$-only protein such as Bid will transiently interact with $\mathrm{Bax}$ and $\mathrm{Bcl}-\mathrm{x}_{\mathrm{L}}$, causing their translocation to the outer mitochondrial membrane. Once inserted into the outer mitochondrial membrane, Bax can form homo-oligomers through $\mathrm{BH} 3$ domain interactions, leading to pore formation and MOMP. Bcl- $x_{\mathrm{L}}$ can bind to $\mathrm{Bax}$ via its $\mathrm{BH} 3$ domain and inhibit homo-oligomerization of Bax, thereby inhibiting MOMP and apoptosis. (Bottom left) The interaction between Beclin and $\mathrm{Bcl}-2$ is disrupted by Nix (during erythroid maturation) or BNIP3 (under low-oxygen conditions), leading to mitophagy. (Bottom right) The locations of Hax, Omi, and Parl (known collectively as the 'HOP complex') are shown. Bax, B-cell lymphoma protein-2-associated X protein; Bcl-2, B-cell lymphoma protein-2; Bcl- $x_{\mathrm{L}}$, Bcell lymphoma protein-2-like- I; BH, B-cell lymphoma protein-2 homology; Bid, BH3-interacting domain death agonist; BNIP3, B-cell lymphoma protein-2/adenovirus EIB I9-kDa interacting protein-3; Hax, HCLSIassociated protein $\mathrm{X}$; HIFI $\alpha$, hypoxia inducible factor-I-alpha; HtrA2/Omi, high-temperature-regulated-A2; MOMP, mitochondrial outer membrane permeabilization; Nix: NIP3-like protein X; Opal, optic atrophy-I; Parl, presenilin-associated rhomboid-like.

studied the other effector, Bak, and how it is triggered by another BH3-only activator, Bid. Using biochemical approaches, they showed that, upon activation, the $\mathrm{BH} 3$ region of Bak becomes exposed and can then insert into a $\mathrm{BH}$ groove-like opening in another activated Bak molecule (whose exposed $\mathrm{BH} 3$ domain seems to bind into the corresponding groove on the first Bak molecule). The scenario that emerges has been described in more detail elsewhere [5]. If an antiapoptotic protein, such as $\mathrm{BCl}-\mathrm{x}_{\mathrm{L}}$, is present, oligomerization of $\mathrm{Bax}$ or Bak is blocked, preventing MOMP. Billen et al. [6] showed that, upon exposure to active Bid, both Bcl- $\mathrm{x}_{\mathrm{L}}$ and Bax insert into outer mitochondrial membranes; $\mathrm{Bcl}-\mathrm{x}_{\mathrm{L}}$ does not form homo-oligomers but binds the $\mathrm{BH} 3$ of $\mathrm{Bax}$ (or presumably Bak), thereby preventing Bax homo-oligomerization and halting the process of MOMP.

The antiapoptotic Bcl-2 proteins, via their $\mathrm{BH}$ grooves, also bind to proteins with functions unrelated to MOMP and apoptosis. For example, the induction of HIF $1 \alpha$ by hypoxia results in the expression of a $\mathrm{BH} 3$-only protein, BNIP3, which displaces the autophagy protein Beclin-1 that is bound to Bcl-2 (via a BH3-like region in Beclin) on mitochondria, and this appears to promote the removal of mitochondria by autophagy [7]. A BH3-only protein closely related to BNIP3, Nix, is also involved in autophagic removal of mitochondria during red blood cell maturation; mice lacking Nix have defective erythrocytes that harbor mitochondria $[8,9]$.

Mitochondria are highly dynamic organelles, routinely undergoing fission and fusion. Coincident with MOMP, mitochondria undergo extensive fission and this has been thought to play an important role in the process of MOMP. However, a recent study [10] showed that while Bcl- $x_{\mathrm{L}}$ effectively blocks MOMP, it does not necessarily prevent fission and therefore the effects are separable. An earlier study [11] had shown that depletion of Bax and Bak from cells, while preventing MOMP, promotes such fission. In light of the results of Billen et al. [6], discussed above, it is tempting to speculate that insertion of Bax and Bak into the membrane, even when oligomerization and MOMP are then neutralized by inserted $\mathrm{Bcl}-\mathrm{x}_{\mathrm{L}}$, nevertheless disrupts an inhibitory effect of the proapoptotic effectors (Bax and Bak) on fission. The relationship between MOMP and mitochondrial fission is further complicated by the finding that novel inhibitors of the fission protein Drp1 are effective inhibitors of MOMP [12].

While much work on MOMP and the mitochondrial pathway of apoptosis focuses on the outside of the mitochondria, events within the mitochondria appear to be involved in the process as well. A recent study [13] examined the disassembly of protein complexes of Opa1, a protein involved in mitochondrial inner membrane remodeling. The BH3-only proteins Bid or Bim, which gain access to the inner membrane space upon Bax/Bak activation and MOMP, appear to perturb Opa1, causing a subtle change in the organization of cristae junctions which facilitates cytochrome c mobilization. Intriguingly, Bak oligomerization and release of cytochrome c, but not Opal disassembly, were blocked by several protease inhibitors in mitochondria exposed to Bid or Bim. In another study [14], genetic ablation of three proteins of the intermembrane space (Hax1, 
HtrA2/Omi, and Parl) were compared. The latter two are proteases, while the former bears two $\mathrm{BH}$ domains (BH1 and $\mathrm{BH} 2$ ) and has previously been suggested to be antiapoptotic. All three knockout animals displayed identical developmental phenotypes, and biochemical investigation showed that Hax1 interacts with Parl to process $\mathrm{HtrA} 2 / \mathrm{Omi}$ to function in a manner required for the survival of Tlymphocytes and some neurons. Thus it seems that factors within the mitochondria may be playing an important role in the response to signals originating from outside of the mitochondria.

\section{Future directions}

Billions of years of evolution have brought us the intricate network of signaling pathways that regulate the life and death of the cell. And while 15 years of research have elucidated the importance of mitochondria for some of these pathways, there is still much to be learned. For example, the relationship between mitochondrial dynamics (that is, fission and fusion) and MOMP is still not fully elucidated, as it appears that there is a relationship between the fission machinery and MOMP, but perhaps this relationship does not involve the process of fission per se. In addition, regulation of MOMP from the inside of the cell is a fairly new area of research that perhaps has more unanswered questions than concrete certainties. Specifically, the relationship, if any, between the 'HOP complex' (Hax1, HtrA2/Omi, and Parl) and Opa1 is not clear. Beyond its function in HtrA2/Omi processing, Parl is thought to function in the cleavage of Opa1, but how this function (and the role of Hax1 and HtrA2/Omi in the process) relates to cellular survival remains obscure. A mutant of Opa1 that resists disassembly was found to block cytochrome c release and apoptosis [13], and it would be interesting to know whether such a mutant would protect cells lacking Hax1, HtrA2/Omi, or Parl. Thus there are still many questions to answer. It has taken us billions of years to reach this point in our story. It may take a few more years to figure out how it all works.

\section{Abbreviations}

A1, B-cell lymphoma protein-2-related protein A1; Bak, B-cell lymphoma protein-2-antagonist/killer-1; Bax, B-cell lymphoma protein-2-associated X protein; Bcl-2, B-cell lymphoma protein-2; Bcl- $\mathrm{x}_{\mathrm{L}}$, B-cell lymphoma protein-2-like-1; BH, B-cell lymphoma protein-2 homology; Bid, BH3-interacting domain death agonist; Bim, B-cell lymphoma protein-2-like-11 (apoptosis facilitator); BNIP3, B-cell lymphoma protein-2/adenovirus E1B 19-kDa interacting protein-3; Drp1, dynaminrelated protein-1; Hax1, HCLS1-associated protein X-1; $\mathrm{HIF} 1 \alpha$, hypoxia inducible factor-1-alpha; HtrA2/Omi,
high-temperature-regulated-A2; Mcl-1, myeloid cell leukemia sequence-1 (B-cell lymphoma protein-2-related); MOMP, mitochondrial outer membrane permeabilization; Nix, NIP3-like protein X; Opa1, optic atrophy-1; Parl, presenilin-associated rhomboid-like.

\section{Competing interests}

The authors declare that they have no competing interests.

\section{Acknowledgments}

MJP is supported by a National Research Service Award postdoctoral fellowship. DRG is supported by grants from the National Institutes of Health and the American Lebanese and Syrian Associated Charities.

\section{References}

I. Bryson B: A Short History of Nearly Everything. New York: Random House, Inc; 2003.

2. Chipuk JE, Green DR: How do BCL-2 proteins induce mitochondrial outer membrane permeabilization? Trends Cell Biol 2008, I 8: 157-64.

3. Gavathiotis E, Suzuki M, Davis ML, Pitter K, Bird GH, Katz SG, Tu HC, Kim $\mathrm{H}$, Cheng $\mathrm{EH}$, Tjandra N, Walensky LD: BAX activation is initiated at a novel interaction site. Nature 2008, 455:|076-8I.

FI000 Factor 4.8 Must Read

Evaluated by Douglas Green with Jerry E Chipuk 29 Oct 200, Atan Gross 7 Nov 2008

4. Dewson G, Kratina T, Sim HW, Puthalakath H, Adams JM, Colman PM, Kluck RM: To trigger apoptosis, Bak exposes its BH3 domain and homodimerizes via $\mathrm{BH}$ :groove interactions. $\mathrm{Mol}$ Cell 2008, 30:369-80

5. Green DR, Chipuk JE: Apoptosis: Stabbed in the BAX. Nature 2008, 455: 1047-9.

6. Billen LP, Kokoski CL, Lovell JF, Leber B, Andrews DW: Bcl-XL inhibits membrane permeabilization by competing with Bax. PLoS Biol 2008, 6:el47.

FI000 Factor 6.0 Must Read

Evaluated by Douglas Green with Jerry E Chipuk 24 Sep 2008

7. Zhang $\mathrm{H}$, Bosch-Marce M, Shimoda LA, Tan YS, Baek JH, Wesley JB, Gonzalez FJ, Semenza GL: Mitochondrial autophagy is an HIF-I-dependent adaptive metabolic response to hypoxia. J Biol Chem 2008, 283:10892-903.

FI000 Factor 3.0 Recommended

Evaluated by Jonathan Gleadle 7 Apr 2008

8. Sandoval H, Thiagarajan P, Dasgupta SK, Schumacher A, Prchal JT, Chen M, Wang J: Essential role for $\mathbf{N i x}$ in autophagic maturation of erythroid cells. Nature 2008, 454:232-5.

FI000 Factor 8.I Exceptional

Evaluated by Richard Youle 13 May 2008, Christoph Borner 27 May 2008, Daniel Klionsky 2I Aug 2008

9. Schweers RL, Zhang J, Randall MS, Loyd MR, Li W, Dorsey FC, Kundu M, Opferman JT, Cleveland JL, Miller JL, Ney PA: NIX is required for programmed mitochondrial clearance during reticulocyte maturation. Proc Natl Acad Sci U S A 2007, 104: | 9500-5. 
10. Sheridan C, Delivani P, Cullen SP, Martin SJ: Bax- or Bak-induced mitochondrial fission can be uncoupled from cytochrome $C$ release. Mol Cell 2008, 31:570-85.

FI000 Factor 4.8 Must Read

Evaluated by Christoph Borner 22 Sep 2008, Vojo Deretic with Isabelle Vergne 24 Oct 2008

II. Karbowski M, Norris KL, Cleland MM, Jeong SY, Youle RJ: Role of Bax and Bak in mitochondrial morphogenesis. Nature 2006, 443:658-62.

FI000 Factor 8.0 Exceptional

Evaluated by David Ginty 24 Oct 2006, Simon Cook 26 Oct 2006

12. Cassidy-Stone A, Chipuk JE, Ingerman E, Song C, Yoo C, Kuwana T, Kurth MJ, Shaw JT, Hinshaw JE, Green DR, Nunnari J: Chemical inhibition of the mitochondrial division dynamin reveals its role in Bax/Bak-dependent mitochondrial outer membrane permeabilization. Dev Cell 2008, I 4:193-204.

FI000 Factor 6.0 Must Read

Evaluated by Barbara Conradt 25 Mar 2008

13. Yamaguchi R, Lartigue L, Perkins G, Scott RT, Dixit A, Kushnareva Y, Kuwana T, Ellisman MH, Newmeyer DD: Opal-mediated cristae opening is $\mathrm{Bax} / \mathrm{Bak}$ and $\mathrm{BH} 3$ dependent, required for apoptosis, and independent of Bak oligomerization. Mol Cell 2008, 31:557-69.

14. Chao JR, Parganas E, Boyd K, Hong CY, Opferman JT, Ihle JN: Haxl-mediated processing of HtrA2 by Parl allows survival of lymphocytes and neurons. Nature 2008, 452:98-102.

FI000 Factor 9.0 Exceptional

Evaluated by Christoph Borner 19 Mar 2008 